\title{
Transforming Occupancy Grids Under Robot Motion
}

\author{
Joris W.M. van Dam ${ }^{1}$ and Ben J.A. Kröse and F.C.A. Groen \\ Faculty of Mathematics and Computer Science, University of Amsterdam \\ Kruislaan 403, NL-1098 SJ Amsterdam, The Netherlands \\ damefri.uva.nl
}

If an autonomous robot uses a local, robot-centered model of its environment, it is essential that this model is transformed every time the robot moves. In our application, an occupancy grid is used to model the robot's environment (see [1]). The transform of occupancy grids involves the computation of numerous intersecting volumes of original and transformed grid cells. If no knowledge is available on the sizes and shapes of the cells, the fastest method to compute these volumes is Monte Carlo estimation. We define a neural network that implements this estimation procedure for all volumes in parallel (see [2]). Each cell of the occupancy grid is represented by a neuron and the transform of the grid is represented by a single layer feed forward network. This paper describes how such a network learns to transform an occupancy grid for a single robot move.

In our network model, neurons $x_{j}^{A}$ in the input layer represent the cells in the original occupancy grid and neurons $x_{i}^{B}$ in the output layer represent the cells in the transformed grid. The activation of each neuron gives the probability of the corresponding cell being occupied. Taking this into account, it can be derived that the activation of the output neurons is given by:

$x_{i}^{B}=1-\prod_{j}\left(1-w_{i j} x_{j}^{A}\right)$

The weights of the network determine the transform and are learned for a given robot move. Starting with a randomly initialised network with each output unit connected to all input units, examples of grids and their correct transforms are generated. With these examples, the weights can be learned using the $\delta$-rule. With the activation function from $(0.1)$, the $\delta$-rule rule gives:

$\Delta w_{i j}=\gamma\left(\hat{x}_{i}^{B}-x_{i}^{B}\right) \cdot x_{j}^{A} \cdot \prod_{k \neq j}\left(1-w_{i k} x_{k}^{A}\right)$

with $\hat{x}_{i}^{B}$ the desired output value taken from the correct transformed grid.

The learning speed of the network is determined by the number of occupied cells (active input neurons) in the learning samples. If we take only few cells $j$ with $x_{j}^{A}=1$, equation (0.2) gives that only few weights are updated. However, if we take too many $x_{j}^{A}=1$, the magnitude of the update will be suppressed by the product term in equation (0.2). On the other hand, we may expect that as learning proceeds, we will get many $w_{i k}=0$ so that the effect of suppression will decrease in time. We defined an optimisation procedure which calculates the optimal number of occupied cells per learning sample as a function of the size of the grid and of the average number $k$ for which $w_{i k} \neq 0$.

Results show that the network is indeed capable of learning the correct weight values. Furthermore, if the number of occupied cells per learning sample is optimised using the procedure mentioned above, the network converges within 4000 iterations for a $256 \times 256$ grid (!!), whereas if no optimisation is performed, the network fails to converge within 20000 learning steps.

\section{References}

[1] Alberto Elfes. Using Occupancy Grids for Mobile Robot Perception and Navigation. Proceedings of the 1989 IEEE Int. Conference on Robotics and Automation.

[2] J.W.M. van Dam, B.J.A. Kröse, F.C.A. Groen, A Neural Network that Transforms Occupancy Grids by Parallel Monte Carlo Estimation. submitted to CSN'93.

\footnotetext{
${ }^{1}$ The investigations were supported by the Foundation for Computer Science in the Netherlands (SION) with financial support from the Netherlands Organization for Scientific Research (NWO) 